\title{
KURZFASSUNG DES EVALUIERUNGSBERICHTS DER UNIVERSITÄT MOZARTEUM SALZBURG
}

\author{
30.04.2004
}

Die Evaluierung der Universität Mozarteum war die erste einer Kunstuniversität in Österreich. Die Grundlage für die Evaluierung bildeten eine im Mozarteum durchgeführte umfangreiche Befragung sowie ein dreitägiger Besuch der internationalen Evaluierungskommission im Mozarteum. Die Kommission führte Gespräche mit Vertretern der Universität (Leitung, Verwaltung, Lehrkörper, Studierenden) aus allen wichtigen Fachgebieten und Organisationseinheiten.

Die Universität Mozarteum verbindet künstlerische, pädagogische und wissenschaftliche Ausbildungen in Musik sowie in darstellenden und gestaltenden Künsten; ihr ausgezeichneter internationaler Ruf ist unbestritten. Diesen Umstand verdankt sie nicht zuletzt den günstigen Faktoren ihrer Lage: der Stadt Salzburg als einem internationalen Kulturmilieu, dem MozartErbe, den Festspielen und den internationalen Sommerkursen. Der tradierte Ruf alleine reicht aber auf Dauer für die zukünftige erfolgreiche Positionierung des Mozarteums in der europäischen Bildungslandschaft nicht mehr aus. Die Ausbildung in den künstlerischen Fächern steht derzeit überall in Europa auf dem Prüfstand; die Konkurrenz wird immer härter, die Musikmärkte verändern sich schnell und die Ressourcen der öffentlichen Hand werden geringer.

So war die Universität Mozarteum wie viele andere entsprechende Ausbildungsinstitute bereits in der jüngeren Vergangenheit mit großen Veränderungen und Herausforderungen konfrontiert. Innerhalb kürzester Zeit sind mehrere Gesetzesänderungen in Kraft getreten, die den Status der Universität Mozarteum, ihre Autonomie und ihre Wirkungsmöglichkeiten betrafen. Auch das Lehrangebot hat Veränderungen durchlaufen. In der Evaluierung kam zum Vorschein, dass die Universität Mozarteum sich zur Zeit in einer schwierigen Anpassungsphase befindet. Die Veränderungen sind noch nicht so lange in Kraft, dass ihre Folgen seriös eingeschätzt werden könnten, aber sie haben naturgemäss Unsicherheiten und Turbulenzen hervorgerufen.

Die externen Gutachter sprechen sich einstimmig für die Beibehaltung des breiten Ausbildungskanons der Universität Mozarteum aus. Die finanzielle Lage ist allerdings besorgniserregend: Die in die Universität investierten knappen finanziellen Mittel ermöglichen zwar zur Zeit noch eine künstlerische und künstlerisch-pädagogische Ausbildung auf insgesamt hohem Niveau, aber schon auf mittlere Sicht ist die Universität unterfinanziert. Sie muss Einsparungen vornehmen bzw. verstärkt Drittmittel einwerben. In diesem Zusammenhang möchte die Kommission daran erinnern, dass der hohe Standard der Universität Mozarteum ein Kapital der Republik Österreich darstellt. In ihren positiven gesellschaftlichen und ökonomischen Auswirkungen auf die Gesellschaft ist die Universität kaum zu überschätzen.

$\mathrm{Zu}$ den positiven Erfahrungen der Evaluierung gehört die von Studierenden vorgebrachte Zufriedenheit mit der Qualität der Lehre. Positiv ist auch, dass die Beschäftigungslage bei Absolventen aller Studienrichtungen sehr gut ist und das sowohl national als auch - besonders bei den Musikern - international. 
Die Universität hat seit 1998 ein erhebliches Raumproblem. Unabhängig von der Lösung der Raumfrage ist die Evaluierungskommission der Ansicht, dass folgende Entwicklungsgebiete für die Universität Mozarteum in der nahen Zukunft von besonderer Bedeutung sind:

- Verbesserung der internen Organisation

- langfristige finanzielle Stabilisierung und Planungssicherheit

- Aufbau eines Systems zur laufenden Qualitätssicherung in Lehre und Studium

- Forschung und Doktoratsstudien, wobei der neue Universitätsstatus maßgebend ist

- Alumnipflege und Fundraising

Zur besseren Bewältigung der Veränderungsphase und der Zukunft empfiehlt die Kommission, dass die Entscheidungsgewalt der Universitätsleitung vermehrt wird.

Der Bericht enthält zahlreiche Empfehlungen für Maßnahmen, die mit diesen Entwicklungsgebieten zusammenhängen. Als Beispiel sei auf die folgenden hingewiesen:

- Organisationsstruktur zwischen den Instituten flexibilisieren

- Aufbau von Strukturen zur Förderung institutioneller und informeller Kommunikation zwischen allen Gruppen

- Einrichtung eines Künstlerischen Betriebsbüros (KBB)

In der Evaluierung wurde festgestellt, dass die Synergievorteile, die sich aus der Breite der Ausbildung ergeben könnten, derzeit nicht genügend umgesetzt werden. Ein wesentliches Hindernis ist dabei die bestehende räumliche Zerstreuung der Aktivitäten. Dennoch ist die Auffassung der Kommission, dass Synergien nicht in erster Linie durch Räumlichkeiten, sondern durch den Willen der Menschen zur Zusammenarbeit entstehen.. Die Entwicklung von diesbezüglichen Visionen und Maßnahmeplänen so wie ihre Verwirklichung würde die Anteilnahme der Lehrer und der Studierenden an ihrer Universität erhöhen. Ein weiterer wichtiger Effekt solcher Aktivitäten wäre die Verbesserung der Sichtbarkeit der Universität in der Öffentlichkeit und ihre Anbindung an die kulturelle Infrastruktur Salzburgs.

Die Umwandlung des Mozarteums zu einer Universität hat diese Institution vor völlig neue Aufgaben und Herausforderungen gestellt, deren Bewältigung viel Entwicklungsarbeit und Zeit in Anspruch nehmen wird. Es muss ein sinnvoller Weg gefunden werden für spezifisch künstlerische Forschung und für Doktoratsabschlüsse im Rahmen einer Kunstuniversität. Die Kommission empfiehlt der Universität Mozarteum zu diesem Zweck Kontakt mit ausländischen Kunstuniversitäten aufzunehmen, wo eine solche Umorganisation ebenfalls im Gang ist oder bereits stattgefunden hat. Insgesamt wird die Universität Mozarteum ermutigt aktiver mit vergleichbaren europäischen Ausbildungsinstituten in Kontakt zu treten. Die Wechselwirkung würde der Entwicklung der Lehre zur Gute kommen.

Auf dem Gebiet der Lehre ergibt sich ein vielschichtiges Gesamtbild. In manchen Bereichen sind Innovationen $\mathrm{zu}$ beobachten, in anderen wäre ein stärkerer Anschluss an die derzeitigen Strömungen von Nöten. Genauere Beobachtungen und Empfehlungen zu den einzelnen Bereichen befinden sich im Bericht. In etlichen Fächern gibt es Bedarf an neuen Professoren und anderen Lehrkräften. Die Kommission betont die Bedeutung von diesbezüglicher Aquise zur Stärkung der Anziehungskraft der Universität Mozarteum für Spitzenkräfte in den künstlerischen Disziplinen wie in der Pädagogik. 
Im Evaluationsbericht wird die Bedeutung der Vorbereitunglehrgänge hervorgehoben. Das Mozarteum ist anziehend für viele ausländische Studenten, aber es müsste im Interesse Österreichs sein besonders auf die nationale Ausbildung in "pre-college"-Stufen zu achten. Die Hochbegabten sollten früh gefunden und in das Wirkungsfeld der Spitzenlehre gebracht werden. Die Vorbereitungslehrgänge des Mozarteums haben bereits in der Vergangenheit wertvolle Arbeit für dieses Ziel geleistet; diese Arbeit soll unbedingt fortgesetzt werden.

Alles in allem muß den Lehrenden und Lernenden sowie der Verwaltung eine sachgemässe Zeitschiene zum Erkennen der Probleme, der Fixierung von Lösungsansätzen und der Umsetzung von Handlungsanweisungen aus dem Evaluationsverfahren gegeben werden. Die Kommission ist der Ansicht, dass nur so die Zukunftsfähigkeit der Universität Mozarteum Salzburg gewährleistet werden kann.

Die Evaluierungskommission wurde geleitet von Fr. Prof. Tuula Kotilainen, Vizerektorin der Sibelius-Akademie 1990-2002, Helsinki. Weitere Mitglieder waren Prof. Björn Boysen, Norges Musikhögskole, Oslo, Prof. Dr. Ulrich Mahlert, Universität der Künste Berlin, Fakultät Musik, Prof. Frans de Ruiter, Königliches Konservatorium Den Haag, Universität Leiden, Dr. Michael Schäfermeyer, Zentrale Bühnen-, Fernseh- und Filmvermittlung der Bundesagentur für Arbeit, Bonn, Prof. Fany Solter, Musikhochschule Karlsruhe und Prof. Dr. Dr. Kurt Zänker, Universität Witten/Herdecke. 

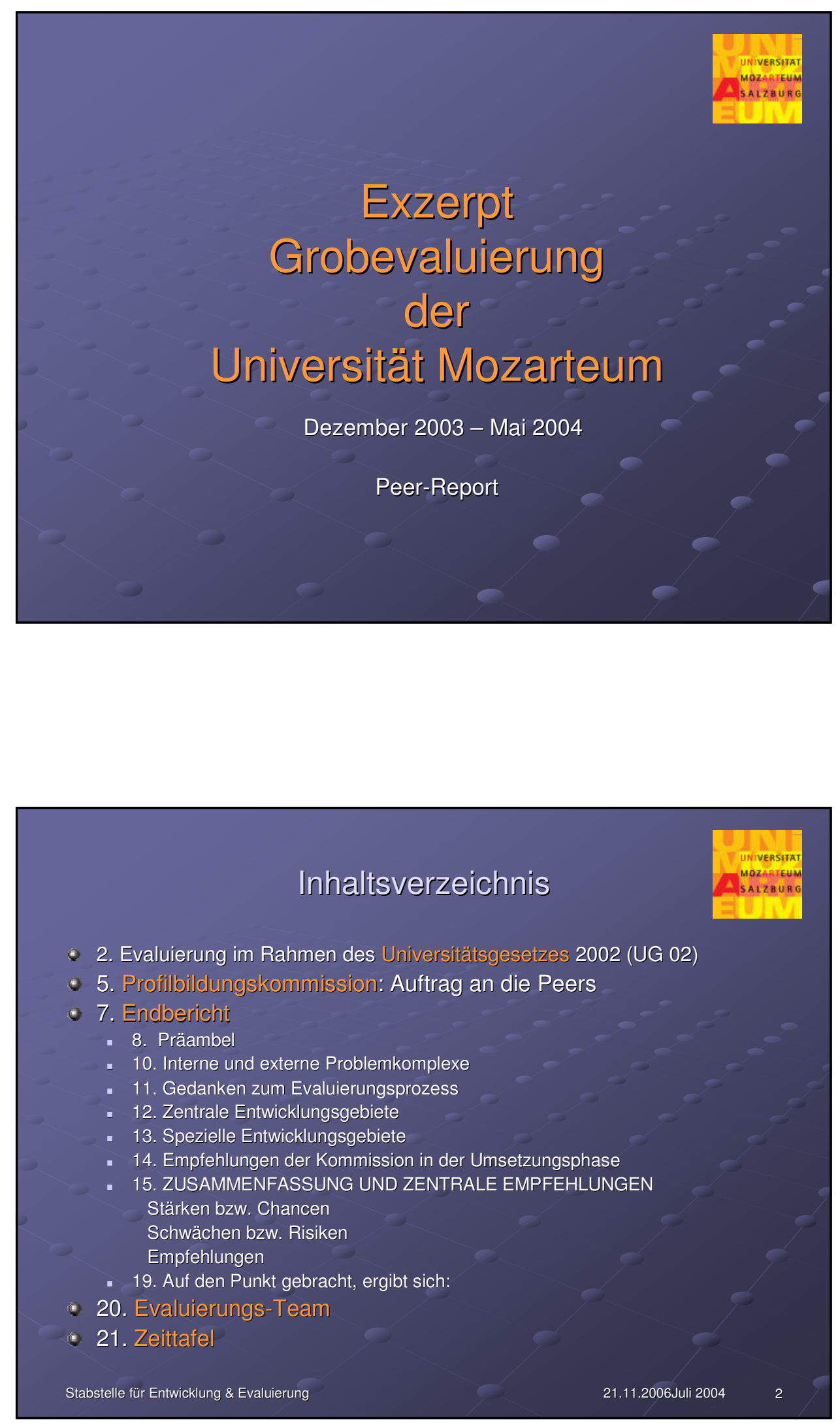


\section{Evaluierung im Rahmen des Universitätsgesetzes 2002 (UG)}

- § 14 UG 2002: Evaluierung und Qualitätissicherung

- (1) Die Universitäten haben zur Qualitäts- und Leistungssicherung ein eigenes Qualitätsmanagementsystem aufzubauen

- (2) Gegenstand der Evaluierung sind die Aufgaben und das gesamte Leistungsspektrum der Universität.

- (8) Die Ergebnisse der Evaluierung sind den Entscheidungen der Universitäitsorgane zugrunde zu legen.

\section{Einbindung der Evaluierung in die rechtlichenhivessina Rahmenbedingungen}

Die Universitätsorgane sind im Wesentlichen

- § 22 UG 2002: Rektorat

- Erstellung eines Entwicklungsplans der Universität

- Erstellung eines Entwurfs der Leistungsvereinbarungen

- Erstellung eines jährlichen Leistungsberichtes, ... und der Wissensbilanz

- § 25 UG 2002: Senat

- Zustimmung zu dem vom Rektorat erstellten Entwurf des Entwicklungsplans (2 Monate). Bei nicht fristgerechter Zustimmung $\Rightarrow$ Weiterleitung des Entwicklungsplans an den Universitätsrat

- \$ 21 UG 2002: Universitätsrat

- Genehmigung des Entwicklungsplan, des Entwurfs der Leistungsvereinbarungen..

- Abschluss des Arbeitsvertrages und der Zielvereinbarungen mit dem Rektor

- § 12 UG 2002: Finanzierung

- Zur Finanzierung der Universität sind Leistungsvereinbarungen mit dem BM:bwk abzuschließen. 2007, 2009, 2011, etc.

- §13 UG 2002: Leistungsvereinbarung

- Leistungsvereinbarung ist ein öffentlich-rechtlicher Vertrag 


\section{Profillbilldungskommission 08.01.2004: Auftrag an das Evaluierungsteam:}

- Einschätzung der Stärken und Schwächen des gegenwärtigen Leistungsangebots des Mozarteums

- Einschätzung der Entwicklungschancen und Risiken der einzelnen Leistungsbereiche und die Zukunftsvorstellungen

- Schwerpunkte des Entwicklungsplans des Mozarteums

- Ressourcenerfordernisse und Einsparungsmöglichkeiten

- Siehe „Peer-Review-Verfahren“ (09.01.2004)

\section{Profillbilldungskommission 08.01.2004: Auftrag an das Evaluierungsteam:}

"Nicht-Ziele":

- Die Evaluationskommission hat sich nicht mit

(a) dem Bau- und Raumbedarf des Mozarteums zu befassen und hat

(b) nicht an dem (eventuell parallel vom Mozarteum erarbeiteten)

Entwicklungsplan mitzuwirken. 


\title{
Evaluationskommission
}

\section{„Endbericht der Evaluierung Unjversität Mozarteum Salzburg"}

\author{
30.04 .2004
}

\section{| Präambel $(s, 4)$}

- Die Universität Mozarteum ist ein breitgefächertes, international renommiertes Ausbildungsinstitut, das künstlerische, pädagogische und wissenschaftliche Ausbildung in Musik sowie in darstellenden und gestaltenden Künsten verbindet.

- Das Mozarteum ist mit seinem drei-Sparten-Modell derzeit auf eine Breitenausbildung konzentriert, aus der sich aber selbststeuernd und selbstorganisierend auch Spitzenkräfte in Musik und Kunst ergeben haben und weiter ergeben werden.

- Das Mozarteum hat seit langem einen sehr guten Ruf. ... 


\section{| Präambel (s.4)}

- Zu bedenken ist, dass die Ausbildung in den künstlerischen Fächern derzeit überall in Europa auf dem Prüfstand steht. Die Konkurrenz wird immer härter, ...

- Das Mozarteum zieht begabte Studenten aus aller Welt an. Es muss jedoch auch wachsam darauf achten, seinen Ruf so wie seine Konkurrenzfähigkeit zu erhalten und weiter zu pflegen, ...

- Ruf und Traditionsverbundenheit des Mozarteums bergen gleichzeitig aber auch Schwächen oder sogar Risiken. ...

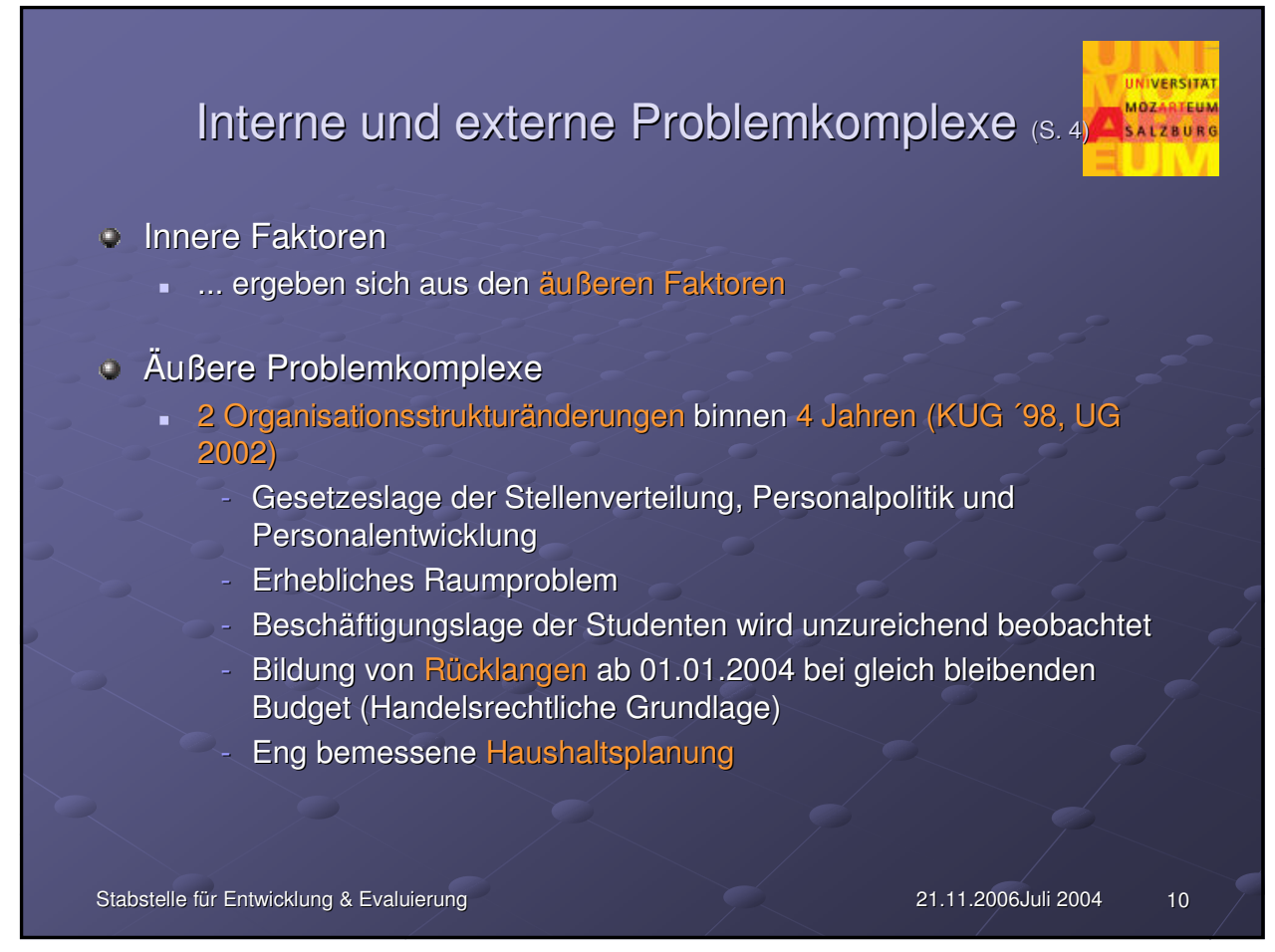


- Pluspunkte

- Immense Arbejtisleistung aller Beteiligten der Universität Mozarteum

- Beantwortung der Selbstbeschreibungsfragebogen durch die Gremien brachten nützliche Erkenntnisse

- Minuspunkte

- Zeitmangel verhinderte:

die Peers, mit eigenen Augen und Ohren Vorstellungen vom musikalischkünstlerischem Niveau zu erlangen,

die Universität, ausgewogene Qualität der Informationsmaterialien (Fragebogen, etc.) zu liefern

Q Wünschenswertes

- Mehr (Selbst-) Einschätzungen an Stelle von (Selbst-) Beschreibungen

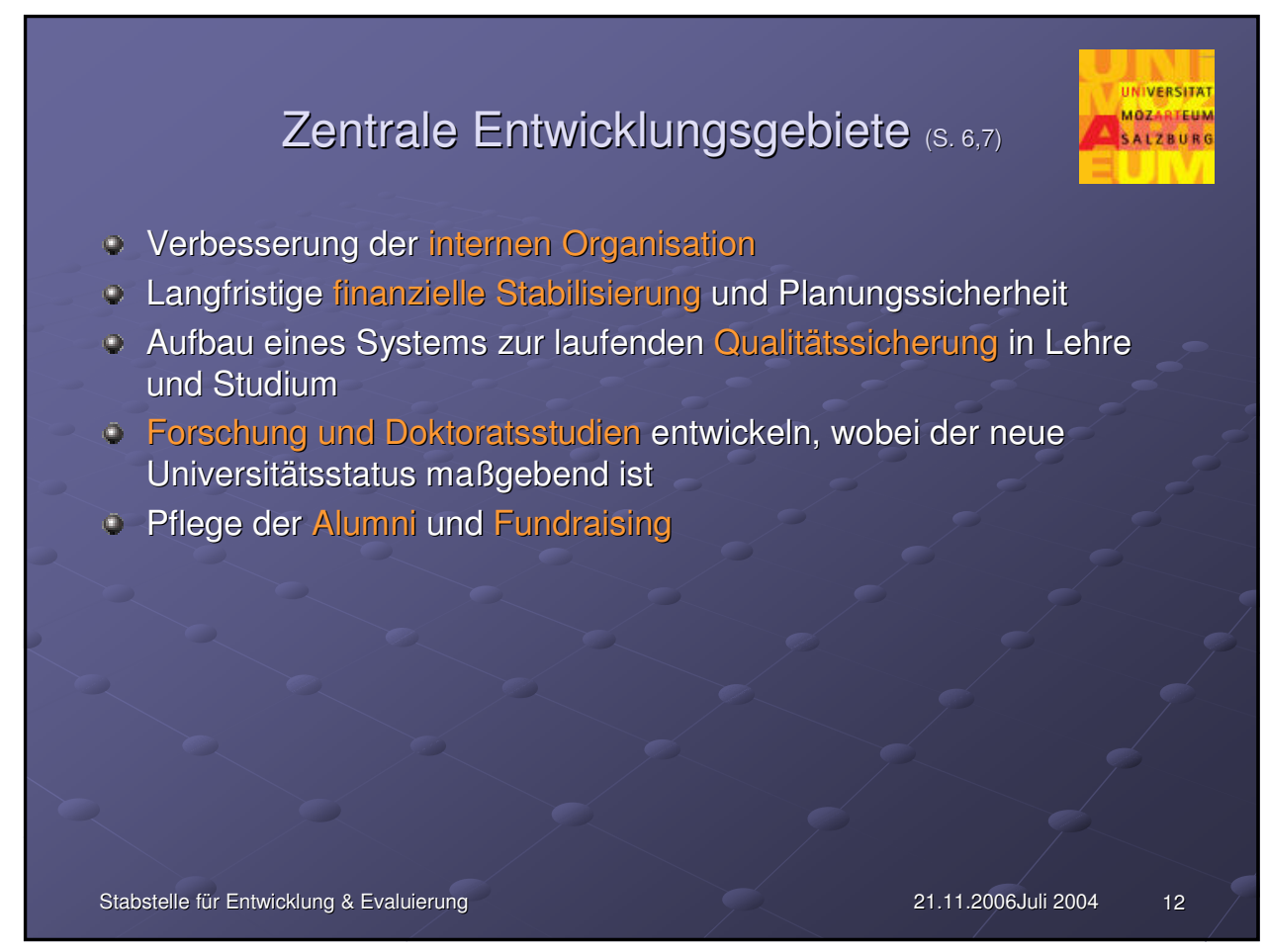




\section{Spezjelle Entwicklungsgebiete $(6,7)$}

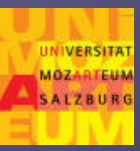

- Synergjien zwischen den unterschiedlichen Sparten und

Instituten/Abteilungen verstärkter ausprägen

- Lehrinhalte in der Musik an das 21. Jahrhundert heranführen

- Altersstruktur des Lehrkörpers

- Nahezu 50 \% Ausländeranteil in der Instrumentalmusikausbildung ist zu überdenken

- Strenge Auswahl der Studierenden (Entwicklung von Steuerungsmaßnahmen im Prüfungswesen, Zulassungsanforderungen innovieren)

- Steigerung der soziale Kompetenzen der Studierenden sowie deren Zufriedenheit

- Raumkonzept entwickeln/umsetzen (Dislocierung)

\section{Empfehlungen der Kommission in der Umsetzungsphase}

* Ausreichend Zeit und Ruhe zur Analyse der Situation und Einleitung der notwendigen Maßnahmen $(5,26)$

- Entwicklungsplan mit messbaren Maßnahmenvorschlägen zu einer nachhaltigen und umfassenden Erneuerung der Struktur (26)

- Interne Nachevalujierung in 1 1/2 bis 2 Jahren zur Klärung der Umsetzung der zentralen Fragen, Beobachtungen und Empfehlungen der Kommission (5)

- Sicherstellung ausreichender Finanzmittel über Sponsoring (26) 


\section{ZUSAMMENFASSUNG UND ZENTRALE EMPFEHLUNGEN: \\ Stärken bzW. Chancen (s. 25)}

- Stärken bzw. Chancen

- Der gute Ruf Mozarteums und die Traditionsverbundenheit

- Die positive Einstellung der Studierenden gegenüber der Lehre

- Größtenteils hochwertiger Unterricht

- Größtenteils gute Beschäftigungschancen sowohl national als auch international

- Eine feste pädagogische und künstlerische Gesamtheit Möglichkeit zu Synergien

\section{Schwächen bzw. Risiken (s. 25)}

- Schwächen bzw. Risiken

- Festharren auf der Tradition und dem Ruf

- Undurchschaubare Organisation (auch auf Grund der vielen Veränderungen)

- Mangelnde Koordination, zu viele überlappende Aktivitäten

- Kommunkationsprobleme

- Räumliche Zerstreuung

- Synergijen werden nicht genügend umgesetzt

- Internationalität wird nicht tiefgehend ausgenutzt

- Zu geringe Kompetenzen des Rektors 


\section{EMPFEHLUNGEN (s. 25)}

- Empiehlungen

- 3-Sparten-Universität aufrechterhalten

- Organisationsstruktur zwischen den Instituten flexibilisieren (Horizontale Organisationsstruktur)

- Erweiterung der Befugnisse des Rektorats; Delegierung der Aufgaben nach klaren, gemeinsam vereinbarten Regeln

- Aufhebung der räumlichen Zerstreuung

- Personalmanagement: Jahres- und Wochenstundenausweitung, Streichung des Kollegiengeldes

- Organisierung des Drittimittelerwerbs

- Aufbau von Strukturen zur Förderung institutioneller und informeller Kommunikation zwischen allen Gruppen

- Interdisziplinäre Institutskonferenz zur Organisation von Synergien

- Synergien zur Qualitätssteigerung auf- und ausbauen

\section{ZUSAMMENFASSUNG UND ZENTRALE EMPFEHLUNGEN (s. 25)}

- Emprehlungen

- Verstärkung der Mitgliedschaft (Teilnahme) von Lehrkräften an universitätsinternen Institutionen

- Einrichtung eines Orchesterrates

- Einrichtung eines Künstlerischen Betriebsbüros (KBB)

- Anfertigung und Realisierung von Strategien in folgenden Bereichen: Verbesserte Außendarstellung der künstlerischen Aktivitäten $\Rightarrow$ Öffentlichkeitsarbeit

Weiterbildung der Lehrkräfte und des für Planung zuständigen Personals (Personalweiterbildung)

Evaluierung der Qualität, Planung der Qualitätssicherung

- Aktive Auseinandersetzung mit den internationalen Lösungen bei Beschäftigten aller Gebiete (Lehre, Verwaltung, Forschung usw.). 


\section{Auf den Punkt gebracht, ergibt sich:}

- Hohe Bedeutung der Universität Mozarteum im In- und Ausland

- Beibehaltung der 3 - Sparten - Universität: Musik - Darstellende Kunst - Bildende Künste

- Ausgewogenheit zwischen Kunst - Wissenschaft - Pädagogik

- Synergien forcieren

- Innovative Rahmenbedingungen gesetzlicher Natur

- Geeignete Räumlichkeiten

- Leistungsanpassung entsprechend europäischer Standards

- Verbesserung der klassische Ausbildung durch zeitgemäße Theorien und Methoden

- Qualifizierung der Forschung

- Steigerung der Kooperationen auf nationaler und internationaler Ebene

- Akkreditierung der Studien

- Auf- und Ausbau eines Qualitätsmanagements

- Effiziente Serviceeinrichtung

\section{Evalujierungs-Team}

- Vorsitz: Frau Professor Tuula Kotilainen, Sibelius Akademie Helsinki

- Herr Prof. Björn Boysen, Norges Musikhögskole, Oslo

- Herr Prof. Dr. Ulrich Mlahlert, Universität der Künste Berlin, Fakultät Musik, Berlin

- Herr Prof. Frans de Rujiter, Königliches Konservatorium Den Haag, Universität Leiden, Den Haag

- Herr Dr. Michael Schäfermeyer, Zentrale Bühnen-, Fernseh- und Filmvermittlung der Bundesagentur für Arbeit, Bonn

- Frau Prof. Fany Solter, Musikhochschule Karlsruhe, Karlsruhe

- Herr Prof. DDr. Kurt Zänker, Universität Witten/Herdecke, Witten 


\section{Zeittafel}

- 18.12.03:

Q 08.01.04:

- 12.01.04:

- 15.01 .05

19.20.21.01.04:

22.01.04:

23.01.04:

- 30.01.04:

16.02.04:

17.02.04:

20.02-15.04.04:

- 20.02.04

- 07. -09.03.04:

- 07.04.04:

- 15.04.04:

6. 30.04.04:

- 07.,11., 19.05.04

- 25.05.05

a. 27.05.04
BM:bwk Wien: Evaluierung zur Prüfung der 3 - Säulen - Universität

BM:bwk. Profilbildungskommission Wien: Mozarteum: Abgabe der Selbstbe-

schreibungsfragebogen (Rektorat, Institute, Studienkommissionen), Peer-

Review-Verfahren, Vorschlagsnamenliste der Peers, mündlicher Antrag zur

Evaluierung. Bm:bwk: Fixierung des Evaluierungsprozesses.

Info-Veranstaltung für alle über Ziele und Maßnahmen der Evaluierung

Aussendung der Fragebongen an IV und Studienkommissionen (Stuko)

Überarbeitung der Fragebogen mit Institutsvorständen und Stuko.

Aussendung der überarbeiteten Fragebogen

Info-Veranstaltung mit Prof. Titscher, Dr. Seitz, Prof. Peter Eckl, Uni PLUS

Fragebogen an Studierende verteilt

Rücklauf der Fragebogen (3 Wochen)

Druck und Versand der FB's an die Peers

Marktanalyse (Pädagogik)

Abgabe „Synopse" = Zusammenfassung der statistische Daten

Begehung der Institute durch die Peers

Treffen der Peers in Frankfurt

Eintreffen des Rohbericht

Eintreffen des Endberichtes mit Kurzfassung: bm:bwk, Mozarteum

Eintreffen der Stellungnahmen

Versandt des Endberichts mit Stellungnahmen: BM:bwk, Land Salzburg

\section{Anmerkungen}

